\title{
Dank an die Gutachter
}

Die Hauptschriftleitung der Zeitschrift Verhaltenstherapie dankt allen Gutachtern und Gutachterinnen herzlich, die durch ihre Reviews im vergangenen Jahr maßgeblich zur Qualität der Zeitschrift beigetragen haben:

Prof. Dr. Borwin Bandelow, Göttingen Prof. Dr. Urs Baumann, Salzburg

Prof. Dr. Peter Becker, Konz

Prof. Dr. Dr. Jürgen Bengel,

Freiburg i.Br.

Prof. Dr. Guy Bodenmann, Fribourg

Prof. Dr. Bernhard Dahme, Hamburg

Prof. Dr. Renate de Jong-Meyer, Münster

Dr. Beate Ditzen, Zürich

Prof. Dr. Manfred Döpfner, Köln

Prof. Dr. Gerhard Goebel,

Prien am Chiemsee

Prof. Dr. Dr. Martin Haerter,

Freiburg i.Br.

Prof. Dr. Kurt Hahlweg, Braunschweig

Prof. Dr. Iver Hand, Hamburg

Prof. Dr. Martin Hautzinger, Tübingen

Prof. Dr. Nina Heinrichs, Bielefeld

Prof. Dr. Beate Herpertz-Dahlmann,

Aachen

Dr. Jörg Heuser, Prien am Chiemsee

Dr. Anja Hilbert, Marburg

Prof. Dr. Wolfgang Hiller, Mainz
Dr. Andrea B. Horn, Fribourg Prof. Dr. Gerhard Lenz, Wien

Prof. Dr. Wolfgang Lutz, Bern

Prof. Dr. Dr. Andreas Maercker, Zürich

PD Dr. Alexandra Martin, Marburg Prof. Dr. Detlev O. Nutzinger,

Bad Bramstedt

Dr. Markus Pawelzik, Münster

Prof. Dr. Franz Petermann, Bremen

PD Dr. Patrick Poessel, Nashville, TN

Prof. Dr. Hans Reinecker, Bamberg

Prof. Dr. Dirk Revenstorf, Tübingen

Prof. Dr. Heinz Rüddel,

Bad Kreuznach

PD Dr. Martin Sack, München

Prof. Dr. Hartmut Schaechinger, Trier

Prof. Dr. Ulrich Schweiger, Lübeck

Prof. Dr. Dr. Hans-Christoph

Steinhausen, Zürich

Prof. Dr. Brunna Tuschen-Caffier,

Freiburg i.Br.

Dr. Heiner Vogel, Würzburg

Dr. Petra H. Wirtz, Zürich 